\title{
Factors Predictive of Weight Gain and Implications for Modeling in Type 2 Diabetes Patients Initiating Metformin and Sulfonylurea Combination Therapy
}

\author{
Jason P. Gordon · Marc Evans · Jorge Puelles · Philip C. McEwan
}

To view enhanced content go to www.diabetestherapy-open.com

Received: July 16, 2015 / Published online: October 7, 2015

(C) The Author(s) 2015. This article is published with open access at Springerlink.com

\section{ABSTRACT}

Introduction: The objectives of this study were to (a) assess the factors associated with weight gain in a population of type 2 diabetes patients escalating from metformin $(\mathrm{M})$ to $\mathrm{M}+$ sulfonylurea $(\mathrm{M}+\mathrm{S})$ and $(\mathrm{b})$ evaluate whether healthcare resource utilization associated with being overweight or obese is underestimated in typical health economic evaluations.

Electronic supplementary material The online version of this article (doi:10.1007/s13300-015-0134-y) contains supplementary material, which is available to authorized users.

J. P. Gordon $(\bowtie)$ · P. C. McEwan

Health Economics and Outcomes Research Ltd, Cardiff, UK

e-mail: jason.gordon@heor.co.uk

J. P. Gordon

Public Health, University of Adelaide, Adelaide, Australia

M. Evans

Llandough Hospital, Diabetes Resource Centre, Cardiff, UK

J. Puelles

Global Outcomes Research, Takeda Development

Centre Europe, TDC (Europe) Ltd, London, UK

P. C. McEwan

Centre for Health Economics, Swansea University, Swansea, UK
Methods: The study was a retrospective cohort study using UK Clinical Practice Research Datalink linked to Hospital Episode Statistics (CPRD/HES) data. The association between baseline phenotypic factors and weight gain was assessed using logistic regression. Hospitalization incidence rates per 1000 person-years for major diabetes-related complications according to body mass index (BMI) at baseline were estimated from the data (observed) and compared to those obtained from a validated diabetes model (predicted).

Results: 11,071 patients were included in the analysis; approximately $40 \%$ gained weight in the first year following escalation to $\mathrm{M}+\mathrm{S}$. Baseline age, HbA1c and gender were found to be predictors of weight gain [odds ratios 0.99 (1-year increment), 1.11 ( $1 \%$ increment) and 0.81 (female vs male), respectively, $p<0.001$ ]. Observed vs predicted incidence rates of hospitalization were 265 vs 13 (normal), 297 vs 31 (overweight), 223 vs 50 (obese) and 378 vs 41 (severe obese).

Conclusion: This analysis suggests there are identifiable patient characteristics predictive of weight gain that may be informative to clinical and economic decision making in the context 
of patients escalating from $\mathrm{M}$ to an $\mathrm{M}+\mathrm{S}$ regimen. Hospital admissions in people with type 2 diabetes were generally under-predicted. A particular focus of future research should be the need for diabetes models to make the likelihood of experiencing an event conditional on BMI.

Funding: Takeda Development Centre Europe Ltd., UK.

Keywords: Diabetes modeling; Therapy escalation; Type 2 diabetes; Weight gain

\section{INTRODUCTION}

The global prevalence of diabetes as of 2013 is estimated at 382 million, accounting for $11 \%$ (548 billion US dollars) of the total global healthcare spend [1]. By 2035, diabetes prevalence is expected to rise by $55 \%$ to 592 million and cost the global economy 627 billion US dollars [1]. The majority of the cost associated with type 2 diabetes is related to the management of diabetes-related macrovascular and microvascular complications, such as cerebrovascular and cardiovascular (CV) disease and diabetic retinopathy, nephropathy and neuropathy [2].

There is an increasing amount of epidemiological evidence relating body mass index (BMI) to increased risk of CV disease and all-cause mortality (ACM) in patients with type 2 diabetes [3, 4]. Importantly, CV and mortality risk equations typically incorporate the effects of elevated BMI indirectly via the inter-relationship between modifiable $\mathrm{CV}$ risk factors (such as cholesterol and systolic blood pressure) and BMI; this approach may underestimate the true morbidity and mortality risk. Consequently, the resource utilization associated with obesity in patients with type 2 diabetes may also be underestimated.
While the importance of avoiding complications that decrease quality of life and consume healthcare resources in people with type 2 diabetes is well understood, the relationship between patient phenotype and resource utilization is less well researched. A recent study by Balkau and colleagues assessed the factors associated with weight gain in type 2 diabetes patients starting insulin [5]: high baseline glycated hemoglobin (HbA1c), insulin dose requirements and lower baseline BMI were all associated with weight gain. In a separate study, van Dieren and colleagues evaluated the relationship between baseline profiles and weight change among participants in the ADVANCE diabetes trial (ClinicalTrials.gov identifier, \#NCT00145925) [6]. They found that baseline factors associated with weight gain were younger age, higher HbA1c, Caucasian ethnicity and number of glucose-lowering medications. These studies illustrate that the identification of phenotypic characteristics that are predictive of weight gain at the time of treatment initiation or escalation may help to develop strategies for avoidance of weight gain [5], and any downstream adverse patient outcomes and excess resource utilization associated with weight gain, among type 2 diabetes patients. Moreover, the accurate prediction of the health and resource consequences associated with the management of type 2 diabetes is crucial to inform decision making in healthcare.

With this in mind, the objectives of this study were to (a) assess the factors associated with weight gain in a population of type 2 diabetes patients escalating to the most common dual oral therapy regimen and (b) evaluate whether healthcare resource utilization associated with being overweight or obese is underestimated in typical health economic evaluations in type 2 diabetes. 


\section{METHODS}

\section{Data}

Consistent with current consensus guidelines on the management of hyperglycemia in patients with type 2 diabetes, metformin (M) augmented with a sulfonylurea $(\mathrm{S})(\mathrm{M}+\mathrm{S})$ is the predominant second-line oral diabetes therapy in clinical practice [7]. The addition of $S$ to $\mathrm{M}$ is known to be associated with increased weight gain [7]. Hence, as a population, this cohort provides a potentially interesting case study to assess the relationship between BMI and healthcare resource utilization.

A retrospective cohort study of linked primary (Clinical Practice Research Datalink, CPRD) and secondary (Hospital Episode Statistics, HES) data informed (a) the baseline profiles for model initialization, (b) baseline patient phenotypic factors predictive of weight gain, and (c) an analysis of observed versus predicted healthcare resource utilization associated with increasing levels of BMI.

The study cohort consisted of all type 2 diabetes patients with a diagnosis code for diabetes (READ/OXMIS code: C10+) between 01/01/2000 and 31/12/2011 in CPRD who initiated treatment with $\mathrm{M}+\mathrm{S} . \mathrm{M}+\mathrm{S}$ combination therapy was defined as "a prescription record for a combination product" or "concomitant use of M or S within 30 days". Index date was defined as the date of addition of $\mathrm{S}$ to $\mathrm{M}$. The following inclusion criteria were applied to obtain the study cohort (Fig. 1): patients escalating from $\mathrm{M}$ monotherapy (to minimize impact of indication bias), to ensure that the beginning of combination therapy was captured accurately (patients were required to have had at least 12 months on $\mathrm{M}$ monotherapy). Patients were required to have at least 3 months' treatment exposure to $\mathrm{M}+\mathrm{S}$ in the post-index period (to minimize bias due to therapy failure). The following exclusion criteria were applied: a prescription for any blood glucose-lowering therapy (other than $\mathrm{M}$ ) prior to the index date. Patients with a diagnosis of malignant disease at any time point prior to the index date, or during the follow-up period.

Data were extracted in quarterly (3-monthly) time periods for the observational period; a 12-month pre-index period was defined for all patients. Data are longitudinal in nature (repeated observations of the same variables and units over periods of time); quarterly measurements were based on the last available record for each study variable. Patient follow-up was restricted to time on $\mathrm{M}+\mathrm{S}$ dual therapy.

Data were extracted based on READ codes and CPRD/HES data files describing patient characteristics: age, sex, smoking status, weight, HbA1c, cholesterol, observed duration of diabetes, in addition to medication use and history of complications (defined in Table 1). Resource utilization data were extracted describing hospitalization and length of stay (LOS).

Studies using CPRD data are covered by ethics approval granted by the Trent Multicenter Research Ethics Committee. This study was granted CPRD Independent Scientific Advisory Committee approval (ISAC protocol number 13-191). This article does not contain any new studies with human or animal subjects performed by any of the authors.

\section{Model}

Assessing the ability of contemporary health economic analyses in type 2 diabetes to capture the resource utilization associated with increasing levels of BMI was undertaken using the IMS CORE Diabetes Model (CDM) [8]. This model has been extensively validated and 


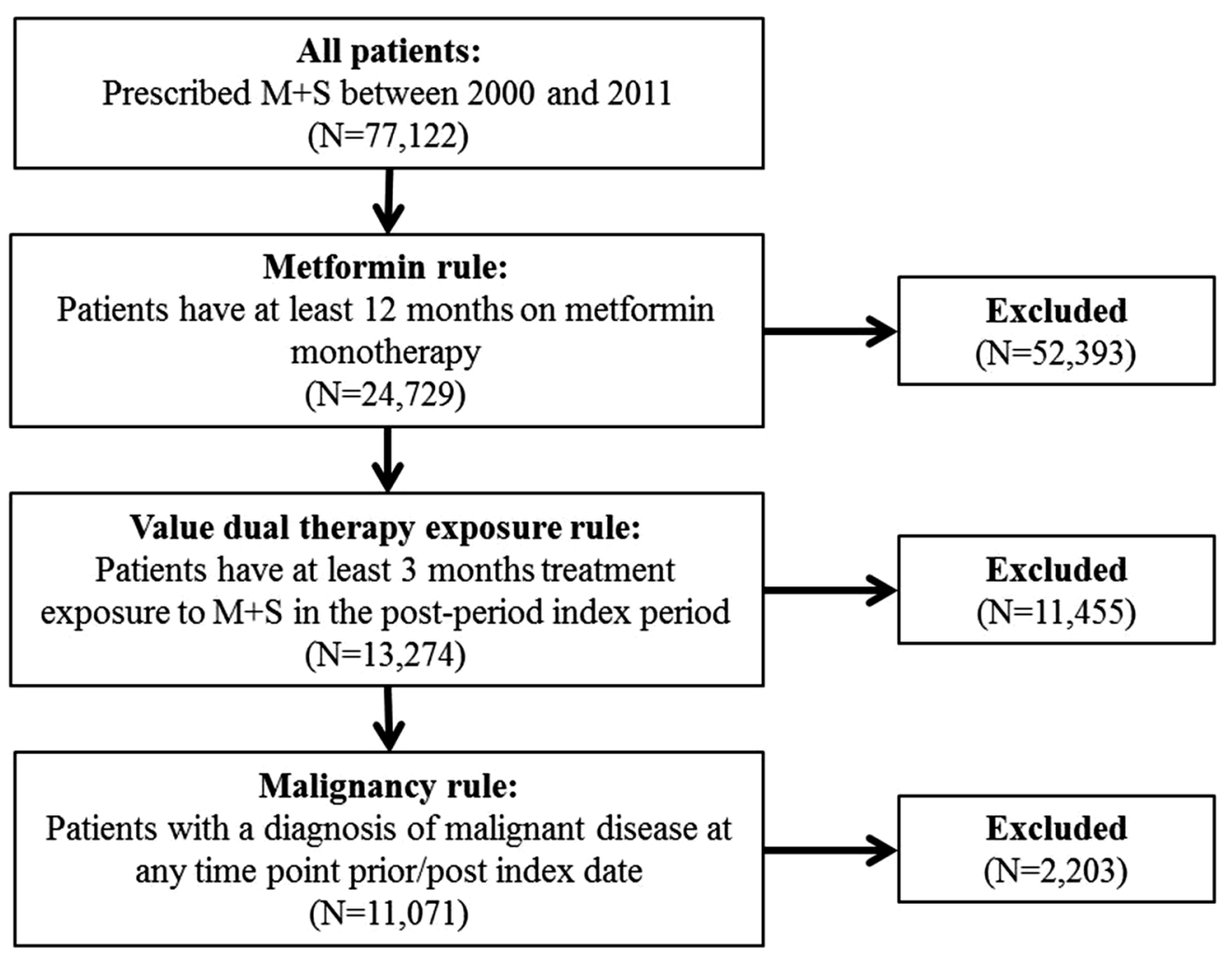

Fig. 1 Flow diagram of study cohort selection with inclusion/exclusion criteria. $M+S$ metformin + sulfonylurea

currently has 87 peer-reviewed publications, predominantly related to cost-effectiveness applications in both type 1 and type 2 diabetes. The model has previously been described in detail [8]. In brief, the model is a fixed-time increment (annual) stochastic simulation using time-, state- and patient-dependent characteristics to model the likelihood of type 2 diabetes-related clinical events occurring. Monte Carlo simulations are performed at the individual patient level using tracker variables to accommodate complex interactions between individual complication sub-models. The CDM simulates the following diabetes-related microvascular and macrovascular complications: angina, myocardial infarction, congestive heart failure, stroke, peripheral vascular disease, diabetic retinopathy, macular edema, cataract, hypoglycemia, ketoacidosis, nephropathy and end-stage renal disease, neuropathy, foot ulcer and amputation, and cardiovascular and non-specific mortality. The CDM incorporates direct and indirect costs, adjusts for quality of life and derives cost-effectiveness and cost-utility estimates.

The model begins by establishing patients' clinical and demographic characteristics, which determines how patients progress through the model. The current analysis using the CDM focuses on the number of events (hospitalizations) and mortality predicted by the model over a 3-year period to facilitate a comparison of observed (CPRD/HES) vs predicted $(\mathrm{CDM})$ hospitalizations and mortality. 
Table 1 Baseline profile by BMI category

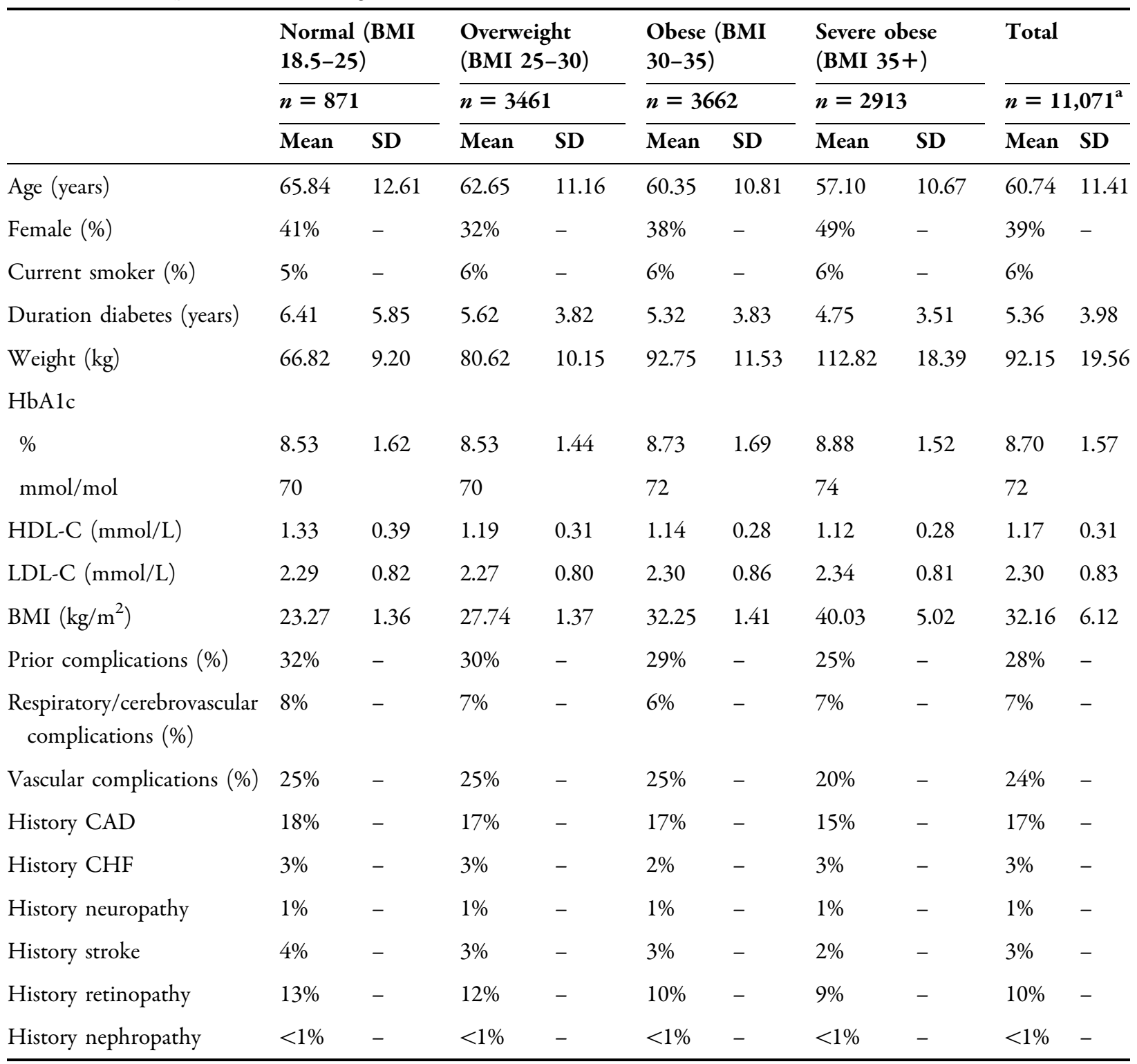

Variable definitions: 'Prior complications' (pre-index history of CAD, CHF, neuropathy, stroke, retinopathy, nephropathy),'Respiratory/cerebrovascular complications' (=asthma, chronic obstructive pulmonary disease, cerebrovascular disease), 'Vascular complications' (=CAD, cerebrovascular disease, peripheral vascular disease, microalbuminuria, chronic kidney disease, retinopathy). History refers to history in 12 -months prior to index date $B M I$ body mass index, $C A D$ coronary artery disease, $C H F$ congestive heart failure, $H b A 1 c$ hemoglobin Alc, $H D L-C$ high density lipoprotein cholesterol, $L D L-C$ low-density lipoprotein cholesterol, $S D$ standard deviation

${ }^{a}$ Overall $n: 10,907$ patients had a BMI record at baseline

\section{Statistical Analysis}

\section{Factors Associated with Weight Gain}

The association between phenotypic factors at baseline (therapy escalation from $\mathrm{M}$ to $\mathrm{M}+\mathrm{S}$ ) and weight gain (defined as $>2 \mathrm{~kg}$ weight change over 12 months following therapy escalation) was assessed in logistic regression analysis with $1=$ weight gain and 0 otherwise. Patients were required to have a valid 
measurement at baseline and 12 months to be included in this analysis, which estimated the probability of weight gain, adjusting for relevant differences in patient characteristics at baseline. Model selection was based on a backwards general-specific methodology, eliminating statistically insignificant covariates, covariate transformations and interaction terms at the 0.05 level of testing to estimate baseline factors predictive of weight gain.

\section{Observed vs Predicted Hospital Admissions}

The association between hospitalizations (number of admissions and LOS) and weight status was assessed using BMI as the weight measurement. This was required to assess the relationship between observed and predicted hospitalizations, as the CDM uses BMI as a risk factor in estimating the incidence of complications. Patients were classified by their BMI at baseline: normal $(\geq 18.5$ and $<25)$, overweight ( $\geq 25$ and $<30$ ), obese $(\geq 30$ and $<35)$, and severe obese $(\geq 35)$.

Observed admissions were compared to the number of events (admissions) predicted by the CDM over a 3-year period, based on the number of patients, baseline demographics and risk factor profile of the cohort. Analysis was stratified by BMI at baseline. The corresponding total and average LOS of admissions from the CPRD data were descriptively summarized.

The observed vs predicted analysis was based on incidence rates to account for variable patient follow-up. For hospitalizations, the risk set was treated as discontinuous risk intervals (i.e., patients were not at risk of a subsequent hospitalization until they had completed the first hospitalization): person-time was calculated by excluding the duration of hospitalizations from the risk set (i.e., follow-up is equal to the sum of the person-time for each risk interval) [9]. Incidence rates of hospitalizations per 1000 person-years were calculated for each BMI category at baseline as the ratio of total number of hospitalizations (numerator) and the total person-years (denominator), multiplied by 1000 . Incidence rates from the CDM were obtained by dividing the predicted number of events by patient follow-up time in the model, multiplied by 1000 .

\section{Observed vs Predicted Mortality}

A comparison between observed and predicted ACM was undertaken based on Kaplan-Meier survival probabilities estimated over a 9-year period for the average patient escalating to $\mathrm{M}+\mathrm{S}$ dual therapy. This comparison aims to assess the predictive performance of the CDM as part of a typical economic evaluation compared to the observed data and provide a second comparative analysis to contrast the analysis of observed vs predicted hospital admissions.

Analyses were undertaken using $\mathrm{R}$ version 2.12.2 (The R Foundation).

\section{RESULTS}

\section{Patient (Baseline) Phenotypic Profiles}

A total of 11,071 patients met the study inclusion/exclusion criteria. The cohort had a mean age at baseline of $60.74(\mathrm{SD}=11.41)$ years, duration of type 2 diabetes of 5.36 $(\mathrm{SD}=3.98) \quad$ years, $\quad \mathrm{HbA} 1 \mathrm{c} \quad$ of $\quad 8.70 \%$ $(72 \mathrm{mmol} / \mathrm{mol})(\mathrm{SD}=1.57)$, weight of $92.15 \mathrm{~kg}$ $(\mathrm{SD}=19.56) \quad$ and $\quad \mathrm{BMI}$ of $32.16 \mathrm{~kg} / \mathrm{m}^{2}$ $(\mathrm{SD}=6.12)$ and were $39 \%$ female (Table 1$)$. There was an increase in baseline mean weight and BMI for increasing BMI categories. Compared to the normal category, the overweight, obese and severe obese categories 
were associated with successively younger mean age at baseline $(65.84,62.65,60.35$, and 57.10 years, respectively) and shorter mean diabetes duration at baseline $(6.41,5.62,5.32$, and 4.75 years, respectively). People in the obese and severe obese categories had higher mean baseline HbA1c than people in the normal category $(8.7$ and 8.9 vs 8.5 , respectively).

\section{Factors Predictive of Weight Gain}

In the 12-month period following initiation of $\mathrm{M}+\mathrm{S}$, weight gain $(1=$ weight gain; 0 otherwise) was observed in $40.39 \%$ of patients with a valid measurement at baseline and 12 months $\quad(n=3139 / 7771) \quad$ and was significantly associated with baseline age $(\mathrm{OR}=0.99$ for 1-year increase in age, $p<0.001)$ female gender $\quad(\mathrm{OR}=0.81$, $p<0.001)$ and baseline HbA1c $(\mathrm{OR}=1.11$ for $1 \%$ increase in HbA1c, $p<0.001$ ) (Table 2).

\section{Observed vs Predicted Hospital Admissions}

Over the 3-year follow-up period, across BMI categories, the most to least common events associated with hospitalizations in the observed dataset (CPRD/HES) were ischemic heart disease (33\%), end-stage renal disease (21\%), congestive heart failure (20\%), myocardial infarction $(14 \%)$, stroke $(13 \%)$, amputation $(<1 \%)$ and blindness $(0 \%)$. The observed incidence rates of hospitalizations per 1000 person-years associated with myocardial infarction, stroke, ischemic heart disease, congestive heart failure, amputation, blindness and end-stage renal disease were 265, 297, 223 and 378 for normal, overweight, obese and severe obese BMI categories, respectively. The predicted (CDM) incidence rates of hospitalizations per
1000 person-years associated with the same complications were 13, 31, 50 and 41 for normal, overweight, obese and severe obese BMI categories, respectively. These data are presented in Fig. 2, which illustrates the observed minus predicted incidence rates were 252, 266, 173 and 337 per 1000 person-years for the increasing BMI categories. Thus, the CDM generally under-predicted the incidence of hospital admissions across event types, except for amputation and blindness, where the model slightly over-predicted incident rates compared to the observed data. Overall, the difference between observed and predicted events was driven by end-stage renal disease, ischemic heart disease, and congestive heart failure hospitalizations, which in absolute terms accounted for $74 \%$ of the observed versus predicted difference $(27 \%, 26 \%, 21 \%$ respectively).

The total LOS associated with the observed (CPRD/HES) 96 hospital admissions in the normal BMI category was 1142 days (mean LOS = 15.0); for the overweight category, total LOS was 4691 (419 admissions, mean LOS $=11.2$; ; for the obese category, total LOS was 4791 (477 admissions, mean LOS $=10.0$ ); and for the severe obese category, total LOS was 3896 (717 admissions, mean LOS $=5.4$ ).

\section{Observed vs Predicted Mortality}

Figure 3 contrasts observed survival from CPRD/HES with predicted survival from the CDM. At year 9, Kaplan-Meier survival probability was 0.780 (95\% CI $0.761-0.800$ ) compared to 0.749 (0.730-0.769). Although the point estimate of the survival probability from the CDM fell below the confidence interval from the observed data, visual inspection of Fig. 3 indicates a reasonable fit to overall mortality. 
Table 2 Baseline factors predictive of weight gain

\begin{tabular}{lllll}
\hline & Odds Ratio & $\mathbf{9 5 \%}$ CI (lower) & $\mathbf{9 5 \% ~ C I ~ ( u p p e r ) ~}$ & $\operatorname{Pr}(>\mathbf{I z I})$ \\
\hline Age (years) & 0.99 & 0.98 & 0.99 & $<0.001$ \\
Gender (female vs male) & 0.81 & 0.71 & 0.91 & $<0.001$ \\
HbA1c (\%) & 1.11 & 1.07 & 1.16 & $<0.001$ \\
\hline
\end{tabular}

$C I$ confidence interval, $H b A l c$ hemoglobin $\mathrm{Alc}, \operatorname{Pr}(>\mathrm{IzI})$ probability being greater than $\mathrm{z}$ and less than $-\mathrm{z}$, where $\mathrm{z}$ is the value of the standard normal distribution

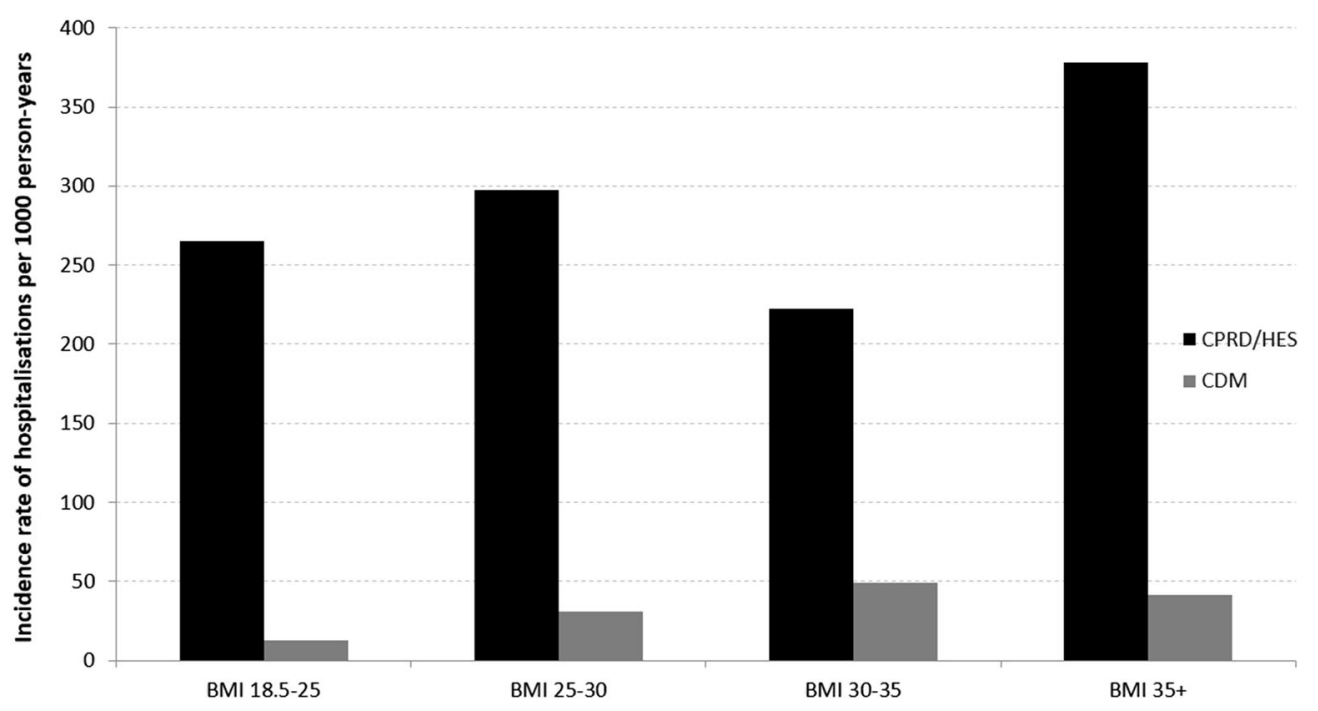

Fig. 2 Comparison of observed incidence rate of hospital admissions stratified by BMI for myocardial infarction, stroke, ischemic heart disease, congestive heart failure, amputation, blindness and end-stage renal disease with

\section{DISCUSSION}

\section{Study Objectives and Results}

The aim of this study was to identify baseline patient characteristics predictive of weight gain and to assess whether typical health economic evaluations capture the excess secondary care healthcare resource utilization associated with weight gain using linked primary and secondary care data from the UK in people with a diagnosis of type 2 diabetes escalating from $\mathrm{M}$ to $\mathrm{M}+\mathrm{S}$ combination therapy.
CPRD/HES compared to the incidence rate predicted by the CDM. BMI body mass index, CDM IMS CORE Diabetes Model, CPRD/HES Clinical Practice Research Datalink/Hospital Episode Statistics

Regarding the first objective of this study, understanding the baseline factors predictive of weight gain is important as it may help to inform both clinical and economic decision making. Where there are identifiable patient characteristics predictive of weight gain, these could help guide treatment decisions and define management strategies, potentially improving patient health outcomes and directing healthcare resources to efficient uses. We found that there are identifiable phenotypic characteristics predictive of weight gain in the $\mathrm{M}+\mathrm{S}$ cohort. Around $40 \%$ of patients gained 


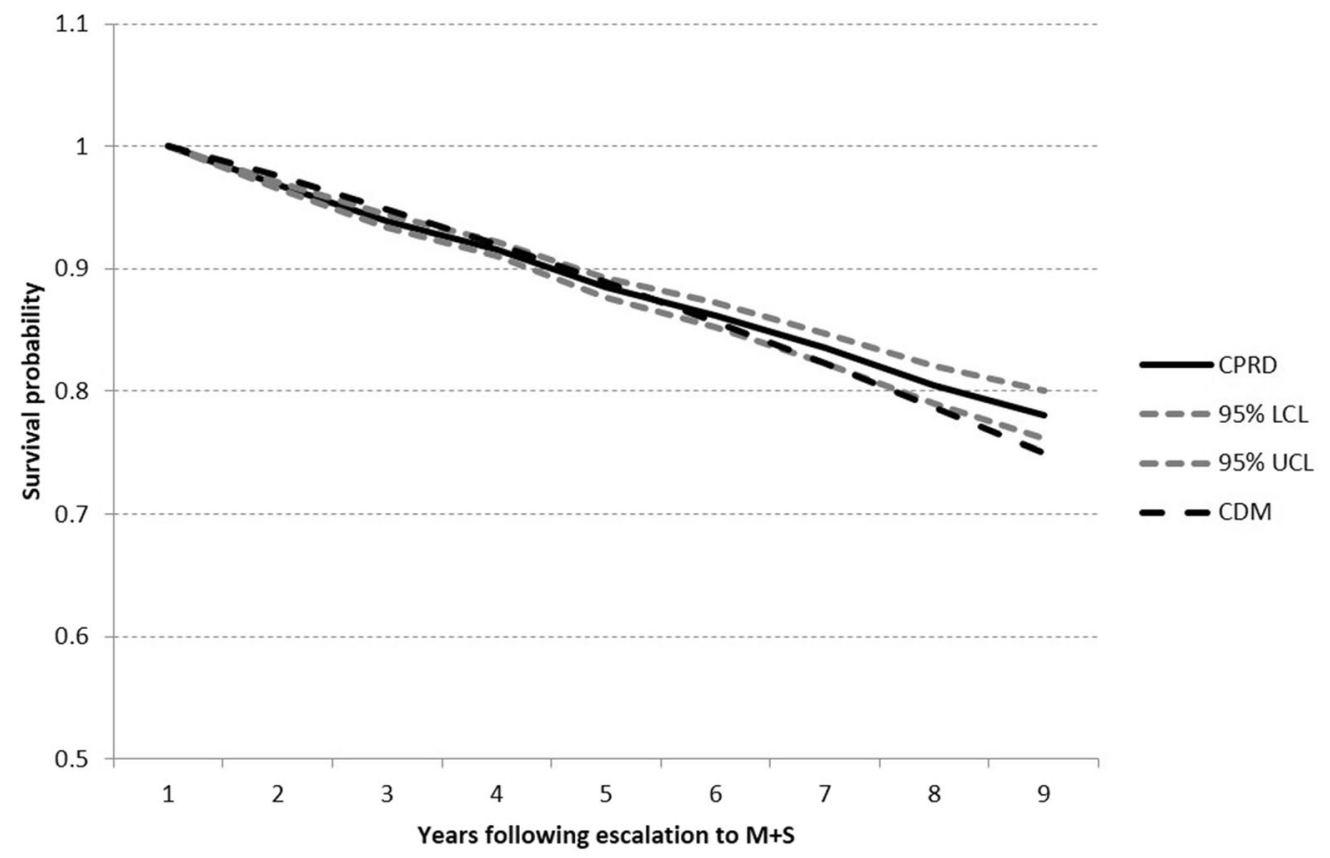

Fig. 3 Comparison of Kaplan-Meier observed survival plot for all-cause mortality (with 95\% upper and lower confidence intervals) compared to output from the CDM. $C D M$ IMS CORE Diabetes Model, CPRD Clinical

weight in the first year following escalation to $\mathrm{M}+\mathrm{S}$; gender, baseline age and baseline HbA1c were found to be predictors. These factors are consistent with the phenotypic factors identified in studies of patients starting insulin therapy [5] and in patients assigned to receive conventional vs intensive management [10]. The results of this study may usefully inform on type 2 diabetes management strategies and investment of healthcare resources; given that $\mathrm{M}+\mathrm{S}$ is the most common second-line therapeutic regimen.

Regarding the second objective of this study, when initialized to the baseline profiles observed in the CPRD population treated with $\mathrm{M}+\mathrm{S}$, the CDM generally under-predicted hospital admissions associated with the most common complications in people with type 2 diabetes. There was an increasing discrepancy between the CPRD observations and CDM
Practice Research Datalink, LCL lower confidence limit, $M+S$ metformin + sulfonylurea, UCL upper confidence limit

predictions for increasing levels of BMI. For instance, the CDM predicted 265 fewer admissions in the normal BMI category, compared to 337 fewer predicted admissions for the severe obese category, per 1000 person-years. This type of discrepancy may have implications for economic modeling used to inform healthcare decisions and clinical decision making where both patient phenotype and the results of economic modeling can impact treatment decisions.

\section{Diabetes Modeling}

It was not the objective of this study to cast doubt over the predictive validity of the CDM. The model has been subject to a number of validation exercises and routinely participates in the Mount Hood challenge meetings, a forum for diabetes modelers to compare and 
contrast model results over a series of standardized validation exercises $[11,12]$; furthermore, the CDM has been shown to capture the incidence of long-term diabetes-related complications with a high degree of accuracy [13]. Our concern rests with whether the risk equations typically used in diabetes models are failing to adequately capture the multiple comorbidities that can occur. The CDM model, as with most published contemporary type 2 diabetes models, is based on the United Kingdom Prospective Diabetes Study (UKPDS) 68 risk equations [14]. BMI features in only one of several risk prediction equations: coronary heart failure. Modeling under-predicted the incidence of myocardial infarction, stroke, ischemic heart disease, congestive heart failure and end-stage renal disease compared to the observed data. The results of this analysis suggest that BMI may have a much larger role to play in determining the risk of diabetes complications, as this analysis suggests higher BMI levels were associated with more hospitalizations. While this study reports an apparent trend of reduced LOS for increasing BMI, overall the findings of this research suggest that secondary healthcare resource utilization is higher for increasing BMI, as reflected by more frequent admission. The recent publication of the updated UKPDS 82 risk equations [15], where BMI is a risk factor for a larger number of diabetes-related complications, further emphasizes the importance of accurately modeling the relationship between complication incidence as a function of increasing BMI.

A consequence of these findings is that long-term projections obtained from diabetes models may not adequately reflect the benefit of improved risk factor profiles. In particular, the value of diabetes management strategies that minimize weight gain may be underestimated as a result. It is noteworthy that the UKPDS equations are most commonly criticized for potentially over-predicting complication rates $[10,16]$; this study suggests they may significantly under-predict total burden associated with diabetes-related complications.

Diabetes models are central to informing decisions around product reimbursement and hence the treatment alternatives available to clinicians and ultimately patients. In this context, and based on the analysis of this patient cohort, there is a case for further investigation around how diabetes models characterize the relationship between weight and hospital admissions; a relationship complicated by many confounding factors such as comorbidities and disease management. Two immediate areas of investigation are how models address the probability of hospitalization as a function of changing BMI and, once hospitalized, the assignment of cost to event. Current models almost exclusively apply an average cost to each type of diabetes complication. This approach may be appropriate where there are no expected differences in hospital LOS as a function of BMI or misleading where LOS is a function of changes in BMI. The current analysis more strongly highlights the need for diabetes models to make the likelihood of experiencing an event conditional on BMI.

\section{Study Limitations}

The findings of this study are set against the following limitations. First, not all relevant confounding factors are captured in the CPRD/ HES databases. Thus, predicting association between BMI and resource use, and baseline factors predictive of weight gain is limited by the coverage and completeness of the data, 
though the databases contain significant detail on the patient characteristics most relevant to this analysis. There may be under-reporting for certain variables within the dataset; for example, the prevalence of congestive heart failure and nephropathy appears low. However, given the age distribution of the cohort, congestive heart failure prevalence around 3\% is not surprising since these patients are probably not that far into their disease course as they have been users of M (first line) and have only just needed augmentation. Nephropathy can take several years to manifest, which may account for the apparently low rate observed; although other studies suggest a significant proportion of type 2 diabetes with a relatively short disease duration would have had nephropathy [17].

Second, by nature, observational studies of this kind will be subject to 'confounding by indication', such that any observed patterns within the data are a function of patient phenotype, and patient phenotype is the reason for prescription of a specific therapy. Restricting analysis to only $\mathrm{M}+\mathrm{S}$ patients minimizes this potential confounder. The inferences that can be made from the current analysis of observed versus predicted hospital admissions are indirectly strengthened by the analysis of observed versus predicted ACM, where estimates from CPRD and the CDM generally predicted the same survival probabilities over a 9-year period. Observed versus predicted survival estimates may have been closer, possibly due to the nature of mortality as an endpoint in that it may be better recorded in CPRD compared to events, and/or the estimation of mortality via UKPDS equations is more accurate; however, the precise reason cannot be determined from this data.
Finally, the CDM was used to evaluate the expected occurrence of events based on risk factor profiles observed in routine clinical practice; these estimates were compared against observed event rates. Statistical adjustment of the data, accounting for the influence of patient characteristics at baseline and over time on the observed relationship between hospitalization rates and BMI, was not undertaken given that the primary aim of this study was to assess the relationship between observed vs predicted hospital admissions in the context of economic evaluation. Thus, reported incidence rates should be interpreted as unadjusted estimates from an epidemiological perspective.

\section{CONCLUSION}

This real-world observational analysis suggests that there are identifiable patient characteristics that are predictive of weight gain, which may be informative to clinical and economic decision making among the context of patients escalating to an $\mathrm{M}+\mathrm{S}$ regimen.

Increased BMI was associated with an increased rate of hospitalization, although average LOS was observed to decrease with increasing BMI. Overall, these findings suggest that there may be an important relationship between increasing BMI and hospitalization that may not be adequately captured in widely used vascular risk equations such as UKPDS. Consequently, the value of diabetes management strategies that minimize weight gain may be underestimated. Models used to predict health outcomes and characterize the value of competing interventions in type 2 diabetes should be investigated in light of these findings. A particular focus of future research should be the need for diabetes models to make 
the likelihood of experiencing an event conditional on BMI.

\section{ACKNOWLEDGMENTS}

The authors thank Beverley Jones (HEOR Ltd.) and Daniel Sugrue (HEOR Ltd.) for writing assistance in the development of this manuscript.

This study was funded by an unrestricted research grant from Takeda Development Centre Europe Ltd., UK.

All named authors meet the International Committee of Medical Journal Editors (ICMJE) criteria for authorship for this manuscript, take responsibility for the integrity of the work as a whole, and have given final approval for the version to be published.

A portion of the findings reported in this article was presented in poster form at 50th European Association for the Study of Diabetes (EASD) Annual Meeting, Vienna, Austria, September 16-19, 2014 and in a poster at the 17th Annual European Congress of International Society for Pharmacoeconomics and Outcomes Research (ISPOR), Amsterdam, The Netherlands, November 8-12, 2014.

Disclosures. JG and PM have served as consultants to and received research funding from Takeda Development Centre Europe Ltd., in relation to this study. JP is an employee of Global Outcomes Research, Takeda Development Centre Europe Ltd. ME has received honoraria and research awards from Novo Nordisk, Sanofi, Novartis, MSD, Takeda and Bayer. JG and PM have received research awards from Boehringer Ingelheim, Takeda Development Centre, Bristol-Myers Squibb, Novo Nordisk, GlaxoSmithKline, Genzyme, Sanofi, Pfizer, AstraZeneca, Otsuka, and Amgen.
Compliance with ethics guidelines. This article does not contain any new studies with human or animal subjects performed by any of the authors. Studies using CPRD data are covered by ethics approval granted by the Trent Multicenter Research Ethics Committee. This study was granted CPRD Independent Scientific Advisory Committee approval (ISAC protocol number 13-191).

Open Access. This article is distributed under the terms of the Creative Commons Attribution-NonCommercial 4.0 International License (http://creativecommons.org/licenses/ by-nc/4.0/), which permits any noncommercial use, distribution, and reproduction in any medium, provided you give appropriate credit to the original author(s) and the source, provide a link to the Creative Commons license, and indicate if changes were made.

\section{REFERENCES}

1. Aguiree $\mathrm{F}$, et al. IDF diabetes atlas, 6th edition. http://www.idf.org/diabetesatlas. Accessed 14 Nov 2014.

2. Hex N, et al. Estimating the current and future costs of type 1 and type 2 diabetes in the UK, including direct health costs and indirect societal and productivity costs. Diabet Med. 2012;29(7):855-62.

3. Prospective Studies Collaboration. Body-mass index and cause-specific mortality in 900000 adults: collaborative analyses of 57 prospective studies. Lancet. 2009;373(9669):1083-96.

4. Eeg-Olofsson K, et al. Risk of cardiovascular disease and mortality in overweight and obese patients with type 2 diabetes: an observational study in 13,087 patients. Diabetologia. 2009;52(1):65-73.

5. Balkau B, et al. (2014) Factors associated with weight gain in people with type 2 diabetes starting on insulin. Diabetes Care. 2014;37(8):2108-13.

6. van Dieren $S$, et al. Weight changes and their predictors amongst 11140 patients with type 2 diabetes in the ADVANCE trial. Diabetes Obes Metab. 2012;14(5):464-9. 
7. Nathan DM, et al. Medical management of hyperglycemia in type 2 diabetes: a consensus algorithm for the initiation and adjustment of therapy a consensus statement of the American Diabetes Association and the European Association for the Study of Diabetes. Diabetes Care. 2009;32(1):193-203.

8. Palmer AJ, et al. The CORE Diabetes Model: projecting long-term clinical outcomes, costs and costeffectiveness of interventions in diabetes mellitus (types 1 and 2) to support clinical and reimbursement decision-making. Curr Med Res Opin. 2004;20(S1):S5-26.

9. Guo Z, Gill TM, Allore HG. Modeling repeated time-to-event health conditions with discontinuous risk intervals: an example of a longitudinal study of functional disability among older persons. Methods Inf Med. 2008;47(2):107.

10. Van Dieren $S$, et al. External validation of the UK Prospective Diabetes Study (UKPDS) risk engine in patients with type 2 diabetes. Diabetologia. 2011;54(2):264-70.

11. Palmer AJ. Computer modeling of diabetes and its complications: a report on the fifth Mount Hood challenge meeting. Value in Health. 2013;16(4):670-85.

12. Mount Hood 4 Modeling Group. Computer modeling of diabetes and its complications: a report on the Fourth Mount Hood Challenge Meeting. Diabetes Care. 2007;30(6):1638-46.
13. Palmer AJ, et al. Validation of the CORE Diabetes Model against epidemiological and clinical studies. Curr Med Res Opin. 2004;20(S1):S27-40.

14. Clarke PM, et al. A model to estimate the lifetime health outcomes of patients with type 2 diabetes: the United Kingdom Prospective Diabetes Study (UKPDS) Outcomes Model (UKPDS no. 68). Diabetologia. 2004;47(10):1747-59.

15. Hayes AJ, et al. UKPDS outcomes model 2: a new version of a model to simulate lifetime health outcomes of patients with type 2 diabetes mellitus using data from the 30 year United Kingdom Prospective Diabetes Study: UKPDS 82. Diabetologia. 2013;56(9):1925-33.

16. Kengne AP, et al. The Framingham and UK Prospective Diabetes Study (UKPDS) risk equations do not reliably estimate the probability of cardiovascular events in a large ethnically diverse sample of patients with diabetes: the Action in Diabetes and Vascular Disease: Preterax and Diamicron-MR Controlled Evaluation (ADVANCE) Study. Diabetologia. 2010;53(5):821-31.

17. Adler AI, et al. Development and progression of nephropathy in type 2 diabetes: the United Kingdom Prospective Diabetes Study (UKPDS 64). Kidney Int. 2003;63(1):225-32. 